\title{
Table des matières
}

Introduction du tome $1 \quad \mathrm{v}$

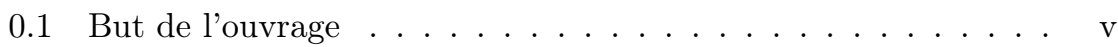

0.2 Contenu de l'ouvrage . . . . . . . . . . . . . . . vi

0.3 Remerciements . . . . . . . . . . . . . . . . . . viii

0.4 Bibliographie sommaire . . . . . . . . . . . . . ix

0.5 Plan structuré du tome $1 \ldots \ldots \ldots \ldots$ xi

I Mécanique quantique et intégrale de chemin 1

1 Rappels de mécanique classique et quantique 3

1.1 Mécanique classique . . . . . . . . . . . . . . 3

1.2 Mécanique quantique . . . . . . . . . . . . . 17

1.3 Mécanique statistique quantique . . . . . . . . . 26

1.4 Notes . . . . . . . . . . . . . . . . . . . . . 33

2 L'intégrale de chemin : introduction $\quad 35$

2.1 Présentation . . . . . . . . . . . . . . . 35

2.2 L'intégrale de chemin pour la particule libre . . . . . . . . . 37

2.3 La particule dans un potentiel $V(q) \ldots \ldots \ldots \ldots \ldots$

2.4 Observables et fonctions de corrélations . . . . . . . . . . 47

2.5 Système quantique à température finie : temps euclidien périodique ................ 54

2.6 L'oscillateur harmonique . . . . . . . . . . . . . . . . . . 61

2.7 Notes . . . . . . . . . . . . . . . . . . . . . . . . . . . . 68

3 Intégrale de chemin et physique statistique $\quad 69$

3.1 Introduction . . . . . . . . . . . . . . . . 69

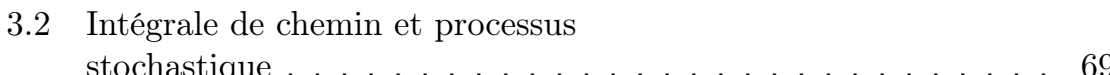

3.3 Mécanique quantique euclidienne et physique statistique $1 \mathrm{D} \ldots \ldots \ldots \ldots \ldots$

3.4 Notes . . . . . . . . . . . . . . . . . . . . . . . . 82 
4 L'intégrale de chemin : présentation générale $\mathbf{8 3}$

4.1 Introduction . . . . . . . . . . . . . . . . . 83

4.2 Systèmes à plusieurs degrés de liberté . . . . . . . . . . . . . . . . . . 83

4.3 Hamiltonien dépendant du temps . . . . . . . . . . . . . . . . . 86

4.4 Méthode du col et limite semi-classique . . . . . . . . . . . . . 87

4.5 Intégrale de chemin dans l'espace de phase . . . . . . . . . . . . 94

4.6 Densité de niveaux et formule des traces . . . . . . . . . . . . . 98

4.7 La particule chargée dans un champ classique . . . . . . . . . . 101

4.8 La particule relativiste . . . . . . . . . . . . . . . 106

5 Systèmes à $N$-corps : bosons, fermions, spin $\mathbf{1 1 5}$

5.1 Intégrale de chemin pour les bosons . . . . . . . . . . . . . 115

5.2 États cohérents et intégrale de chemin pour le spin . . . . . . . . . . . . . . . . . . 136

5.3 Fermions, algèbre de Grassmann et intégrale de chemin anti-commutante . . . . . . . . . . . . . 144

5.4 Conclusion : avantages et désavantages de l'intégrale de chemin 153

5.5 Notes . . . . . . . . . . . . . . . . . . . 155

II Théorie des champs et intégrale fonctionnelle 157

6 L'intégrale fonctionnelle : le champ libre 159

6.1 Introduction . . . . . . . . . . . . . . . . . . . . 159

6.2 Le champ libre comme limite continue du modèle gaussien . . . 160

6.3 La fonction de corrélation à deux points et le propagateur . . . . . . . . . . . . . . . . . 173

6.4 Fonctions de corrélation à $N$ points et théorème de Wick . . . . . . . . . . . . . . . . . . . . . . . . 180

6.5 Configurations du champ libre gaussien . . . . . . . . . . . 183

6.6 Opérateurs composites et développement à courte distance $(\mathrm{OPE}) \ldots \ldots$. . . . . . . . . . . . . . . . 187

6.7 Équations quantiques du mouvement (Schwinger-Dyson) . . . . . . . . . . . . . . . . 199

6.8 Champ libre, particule relativiste et marches aléatoires . . . . . . . . . . . . . . . . . 201

6.9 Du champ scalaire aux bosons non relativistes .................. . 203

6.10 Notes ....................... . . . 204

7 La théorie des champs $\phi^{4}$ : théorie des perturbations $\quad 207$

7.1 Introduction . . . . . . . . . . . . . . . . . . . 207

7.2 Intégrale fonctionnelle pour la théorie $\phi^{4}$. . . . . . . . . . . . . 208

7.3 Le développement perturbatif : diagrammes de Feynman . . . . 212

7.4 Fonctions de corrélations et diagrammes connexes . . . . . . . . 226 
7.5 Diagrammes et amplitudes irréductibles . . . . . . . . . . . 231

7.6 L'action effective $\Gamma[\varphi] \ldots$. . . . . . . . . . . . . . . . . . . . 238

7.7 Calcul des amplitudes de Feynman . . . . . . . . . . . . . . . 249

7.8 Équations de Schwinger-Dyson . . . . . . . . . . . . . . . 258

7.9 Symétries, courants conservés et théorème de Noether . . . . . 259

7.10 Notes . . . . . . . . . . . . . . . . . . . . . 264

8 La théorie $\phi^{4}$ : Renormalisation à l'ordre d'une boucle $\mathbf{2 6 5}$

8.1 Introduction . . . . . . . . . . . . . . . 265

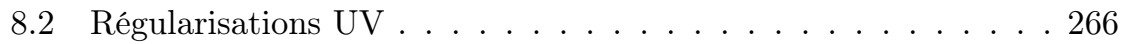

8.3 Les divergences UV en $D=4$. . . . . . . . . . . . . . . 274

8.4 Renormalisation de $\phi^{4}$ à $D=4$ : principe . . . . . . . . . . . 278

8.5 Renormalisation de la théorie de masse nulle à $D=4$. . . . . 283

8.6 Renormalisation de la théorie massive pour $D=4$. . . . . . . . . . . . . . . . . . . . . . . 286

8.7 Échelle de renormalisation et couplages effectifs ....................... . . 288

8.8 Transformations d'échelle et groupe de renormalisation ................... . . 294

8.9 Renormalisation de $\phi^{4}$ en dimension $D<4$. . . . . . . . . . 299

8.10 Analyse des flots du groupe de

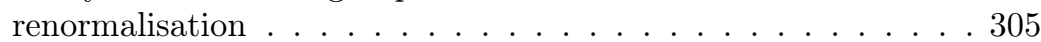

8.11 Renormalisation dimensionnelle . . . . . . . . . . . . 311

8.12 Notes . . . . . . . . . . . . . . . . . . . 312

9 Renormalisation perturbative : aperçu général 313

9.1 Introduction . . . . . . . . . . . . . . . . . . 313

9.2 Divergences UV et comptage de puissance . . . . . . . . . . . 313

9.3 Renormalisation et contretermes . . . . . . . . . . . . . . 322

9.4 Premier aperçu historique . . . . . . . . . . . . . . . . . 325

9.5 Notes . . . . . . . . . . . . . . . . . . . 328

$\begin{array}{lr}\text { Index } & 329\end{array}$

$\begin{array}{ll}\text { Bibliographie } & 334\end{array}$ 